\title{
A Modelagem Matemática em contextos da Educação Estatística: uma proposta de atividade direcionada ao Ensino Médio
}

\author{
Mathematical Modeling in Statistical Education contexts: a proposal for an activity aimed at \\ Secondary Education \\ Modelización Matemática en contextos de Educación Estadística: una propuesta para una \\ actividad dirigida a la Educación Secundaria
}

Recebido: 29/12/2021 | Revisado: 03/01/2022 | Aceito: 10/01/2022| Publicado: 14/01/2022

Francisco Neto Lima de Souza

ORCID: https://orcid.org/0000-0001-5340-8630 Instituto Federal de Educação, Ciência e Tecnologia do Rio Grande do Norte, Brasil

E-mail: francisco.neto.105@ufrn.edu.br

Marconio Silva dos Santos

ORCID: https://orcid.org/0000-0002-3756-6468

Universidade Federal do Rio Grande do Norte, Brasil

E-mail: marconio.santos@ufrn.br

\begin{abstract}
Resumo
O trabalho ora apresentado compreende uma aula inovadora do componente curricular Matemática que propõe um estudo da Estatística mediante uma abordagem de ensino que tem como fio condutor a Modelagem Matemática. Objetiva desenvolver o tratamento numérico, analítico e crítico de dados estatísticos a partir das informações veiculadas pelas plataformas CoronavírusRN e RN+vacina. O estudo está norteado pela seguinte questão focal: como explorar as informações presentes nas plataformas CoronavirusRN e RN+Vacina a partir de um ambiente de Modelagem Matemática para ensinar Estatística por meio de uma aula inovadora. Como resultado, apresentamos uma sequência didática composta por três momentos, a saber: experimentação (1), criação e resolução de um modelo (2), validação e modificação do modelo (3). O protótipo de aula é direcionado a alunos do $3^{\circ}$ ano do ensino médio.
\end{abstract}

Palavras-chave: Ensino de matemática; Ensino de estatística; Modelagem matemática; Covid-19.

\begin{abstract}
The work presented here comprises an innovative class in the Mathematics curricular component that proposes a study of Statistics through a teaching approach that has as its guiding principle the Mathematical Modeling. It aims to develop the numerical, analytical and critical treatment of statistical data from the information provided by the CoronavirusRN and $\mathrm{RN}+\mathrm{vaccine}$ platforms. The study is guided by the following focal question: how to explore the information present in the CoronavirusRN and RN+Vaccine platforms from a Mathematical Modeling environment to teach Statistics through an innovative class. As a result, we present a didactic sequence composed of three moments, namely: experimentation (1), creation and resolution of a model (2), validation and modification of the model (3). The class prototype is aimed at $3 \mathrm{rd}$ year high school students.
\end{abstract}

Keywords: Mathematics teaching; Teaching of statistics; Mathematical modeling; Covid-19.

\section{Resumen}

El trabajo aquí presentado comprende una clase innovadora del componente curricular de Matemáticas que propone un estudio de la Estadística a través de un enfoque de enseñanza que tiene como principio rector el Modelado Matemático. Tiene como objetivo desarrollar el tratamiento numérico, analítico y crítico de los datos estadísticos a partir de la información que brindan las plataformas de vacunas CoronavirusRN y RN +. El estudio se guía por la siguiente pregunta focal: cómo explorar la información presente en las plataformas CoronavirusRN y RN + Vaccine desde un entorno de Modelado Matemático para enseñar Estadística a través de una clase innovadora. Como resultado, presentamos una secuencia didáctica compuesta por tres momentos, a saber: experimentación (1), creación y resolución de un modelo (2), validación y modificación del modelo (3). El prototipo de la clase está dirigido a alumnos de $3^{\circ}$ de bachillerato.

Palabras clave: Enseñanza de las matemáticas; Enseñanza de la estadística; Modelo matematico; Covid-19. 


\section{Introdução}

No currículo da Matemática, a Estatística é um tema relativamente novo. Apesar da notória importância, quando se observa as abordagens usuais desse tópico nas escolas, vê-se na Estatística uma aproximação à aspectos procedimentais e mecânicos, resumida, muitas vezes, ao cálculo da média, moda e mediana. Ponte, Brocado e Oliveira (2019) colocam que, diante disto, ela vem se tornando um dos assuntos mais maçantes de se ensinar e se aprender, enfatizando a mecanização e a memorização, fatores esses, que juntos, não agem no sentido de facilitar o desempenho e mostrar a importância desse conteúdo aos alunos.

Na contramão desse modelo de ensino, este trabalho buscou redimensionar a ação educativa a partir do propósito de que os estudantes sejam capazes de pensar a sua realidade de forma a construir habilidades para agirem como sujeitos autônomos e transformadores (Freire, 2014). Surge, assim, uma proposta de aula na forma de um conjunto de atividades interconectadas (sequência didática) pensadas para a $3^{\circ}$ série do ensino médio, cujo foco é o estudo da Estatística em um ambiente marcado pela Modelagem Matemática (MM).

Barbosa (2004) coloca que a MM constitui um "ambiente de aprendizagem onde os alunos são convidados a indagar e/ou investigar, por meio da matemática, situações oriundas de outras áreas da realidade” (p. 6), permitindo assim, a constituição de um pensamento interdisciplinar mediado pela Matemática e capaz de fazer com que o aluno possa refletir a sua realidade criticamente.

Meneguelli (2017) coloca que a MM cria “[...] condições para os estudantes pensarem estatisticamente, formulando hipóteses e elaborando estratégias de validação dessas hipóteses" (p. 26). Com isso, ao propor problematização, investigação, questionamento, reflexão e diálogo, as atividades pautadas na MM favorecem a construção de uma abordagem que possibilita o trabalho com a Estatística.

A temática central para o desenvolvimento das atividades foi a Covid-19¹. Essa escolha se deu, pois, a pandemia do novo coronavírus, causador da doença, tem tomado conta das reportagens, noticiários e revistas, particularmente, pelos cenários preocupantes que estão se agravado, originando problemas estruturais que podem ser observados em vários lugares do Brasil e do mundo.

Nos meios de comunicação, os gráficos, enquanto instrumentos estatísticos, estampam a capa de revistas e ganham vários minutos em jornais televisivos, mostrando a importância do desenvolvimento da habilidade de leitura e interpretação dos dados. No mais, a Estatística vem fazendo a diferença na sociedade e se mostrando uma ferramenta imprescindível à organização, síntese e apresentação dos dados da pandemia.

Acompanhando uma tendência mundial e objetivando dar transparência ao processo de gestão da pandemia no estado do Rio Grande do Norte, o Laboratório de Inovação Tecnológica em Saúde da Universidade Federal do Rio Grande do Norte (LAIS/UFRN), em parceria com outras instituições e núcleos de pesquisa criaram o Ecossistema Tecnológico de Combate à Covid-192. Essa plataforma reúne dados sobre a gestão da pandemia e disponibiliza cursos para capacitar os profissionais da saúde, serviços de teleconsultoria e dados de prestação de contas. Neste trabalho, dá-se destaque a duas dessas. A primeira é a plataforma CoronavírusRN ${ }^{3}$, um painel de informações que contém todos os dados oficiais sobre a pandemia no RN, e a segunda é a plataforma $\mathrm{RN}+\mathrm{vacina}^{4}$, criada para administrar as doses das vacinas e servir como um instrumento de transparência do processo de gestão de aplicações.

\footnotetext{
${ }^{1}$ Covid-19 é a doença causada pelo novo Coronavírus. A sigla surge da combinação de dois termos, covid e 19 . O termo covid é uma abre viação para corona virus disease, ou seja, doença do coronavírus e o algarismo 19 faz referência ao ano em que os primeiros casos da doença foram registrados.

${ }^{2}$ Ecossistema Tecnológico de Combate à Covid-19 é uma plataforma criada a partir de vários colaboradores que integram diferentes sistemas de informação, com o intuito de reunir informações sobre a pandemia e usar os análise de dados em saúde nas ações de preparo, vigilância e resposta a surtos epidemiológicos no Rio Grande do Norte (Valentin, 2021).

3 https://covid.lais.ufrn.br/

${ }^{4}$ https://rnmaisvacina.lais.ufrn.br/cidadao/
} 
A respeito de plataformas dessa natureza, a Base Nacional Comum Curricular (BNCC) sinaliza que elas podem promover um ambiente potencialmente rico para pensar estatisticamente, oportunizando a reflexão sobre os procedimentos e métodos estatísticos (Brasil, 2018). Com isso, têm-se à disposição ferramentas que podem fomentar situações de aprendizagem, principalmente pela diversidade de formas com que as informações são apresentadas, constituindo-se como um ambiente rico para o desenvolvimento do pensamento estatístico.

Considerando a prática docente e a preocupação com o ensino de Matemática, mais precisamente o ensino da Estatística, chega-se a seguinte questão-foco que norteia a construção deste trabalho: como explorar as informações presentes nas plataformas CoronavirusRN e RN+Vacina a partir de um ambiente de Modelagem Matemática para ensinar Estatística por meio de uma aula inovadora 5 ?

Levando em consideração a questão focal deste estudo, o objetivo geral do trabalho é apresentar uma aula inovadora que busca desenvolver o tratamento numérico, analítico e crítico de dados estatísticos a partir das informações veiculadas pelas plataformas CoronavírusRN e RN+vacina, ambas administradas pelo LAIS/UFRN.

Haja vista tal anseio, foram elaborados quatro objetivos específicos, intimamente relacionados, para que o objetivo geral posto seja alcançado, a saber: avaliar informações presentes em gráficos de modo a produzir considerações fundamentadas em dados; entender o que é e qual o significado de alguns termos epidemiológicos utilizados no processo de investigação estatística da pandemia; produzir um modelo matemático que estime o número de vacinados a partir do tempo; fomentar o desenvolvimento da literacia, do raciocínio e do pensamento estatísticos.

\section{Revisão Bibliográfica}

A Educação Estatística (EE) segundo Campos, Wodewotzki e Jacobini (2011) é um campo de trabalho que pensa o ensino da Estatística e valoriza as "práticas Estatísticas aplicadas a problemática do cotidiano do aluno que, com a ajuda do professor, toma consciência de aspectos sociais muitas vezes despercebidos, mas que nele (cotidiano) se encontram fortemente presentes" (p. 12). Nesse sentido, ela propõe uma linha de trabalho que trata a lógica em conexão com o contexto, tendo três competências fundantes: a literacia, o raciocínio e o pensamento estatístico.

Os mesmos autores colocam que a literacia Estatística se refere "ao estudo de argumentos que usam a Estatística como referência, ou seja, à habilidade de argumentar usando corretamente a terminologia estatística" (p. 23). Compreende a manipulação dos conhecimentos estáticos que possibilitam ler, interpretar, analisar e avaliar uma informação estatística de forma que se possa tomar decisões e fazer asserções sobre fatos.

O raciocínio estatístico é a forma com que as pessoas raciocinam os dados estatísticos, ou seja, estamos tratando da forma como o sentido é dado a informação (Barbosa, 2014). Segundo Lopes e Fernandes (2014), "este envolve fazer interpretações adequadas com base nos conjuntos de dados, fazer representações de dados e resumos estatísticos e pode envolver fazer conexões entre os conceitos envolvidos e combinar ideias sobre os dados” (p. 72). Dessa forma, pensa-se em fomentar um espaço que tem como principal objetivo trabalhar os conceitos próprios da Estatística.

O último componente da tríade fundante da EE é o pensamento estatístico que, segundo Assis (2015), é "um olhar geral para o problema apresentado, uma capacidade de questionar com espontaneidade a realidade observada a partir da estatística" (p. 27). Dessa forma, é valorizado o contexto no qual os problemas estão inseridos, para que se possa pensar o que os dados querem informar sobre dada situação, tendo noção da dimensão completa do problema.

\footnotetext{
${ }^{5}$ A aula inovadora, no âmbito desse trabalho, pode ser entendida como uma proposta didática que ainda não foi vivenciada pelo professor e pelo aluno, fugindo um pouco da forma como o conteúdo é ministrado tradicionalmente e se constituindo como uma via para a compreensão do conteúdo com mais significado.
} 
Cabe observar que o ensino da Estatística deve ser pensado a partir dessas três competências, haja vista que estão intimamente relacionadas. A esse despeito, é muito bem colocado por Santana (2018) que “[...] estas três dimensões da EE não são excludentes. Ao contrário, intercalam-se e se complementam mutuamente, pois seu objeto de investigação teórico e concreto dialoga continuamente com a práxis social tanto dos acadêmicos quanto dos professores" (p. 18). Como a Estatística é tratada enquanto um assunto do rol de conteúdos da Matemática, vê-se uma valorização de procedimentos mecânicos e a ausência de contexto nos dados apresentados. Percebe-se que essa forma de trabalho não favorece o desenvolvimento articulado da literacia, do pensamento e do raciocínio estatísticos, de modo a propiciar uma aprendizagem com significado (Meneguelli, 2017).

Mendonça e Lopes (2011) assim como Campos et al. (2011) afirmam que um ambiente permeado pela MM é potencialmente rico para o desenvolvimento dessas três competências de forma conjunta, pois a MM, enquanto uma via para o ensino, fomenta o desenvolvimento do pensamento matemático, permitindo que o aluno participe ativamente do seu aprendizado a partir de situações reais que são investigadas, questionadas e conjecturadas na tentativa de entender melhor a realidade que o cerca.

Segundo Bertone et al. (2014), pode-se entender a MM enquanto uma área de investigação dentro da Educação Matemática que "consiste na arte de transformar problemas da realidade em problemas matemáticos e resolvê-los, interpretando suas soluções na linguagem do mundo real" (p. 18). Dessa forma, são observadas situações da realidade com o intuito de descrevê-las matematicamente, objetivando criar modelos capazes de traduzir o seu comportamento.

O potencial multidisciplinar e investigativo inerente a MM faz com que Barbosa (2003) a conceitue como "um ambiente de aprendizagem em que os alunos são convidados a problematizar e investigar situações com referência em outras áreas da realidade" (p. 69). O fato é que, com essa metodologia é oferecida ao aluno uma oportunidade para criar, construir e analisar as relações entre a Matemática e o mundo real, de forma que ele possa se apropriar dos conceitos, enquanto age com autonomia dando significado a ideias matemáticas (Miguel \& Natti, 2008).

Algumas experiências exitosas com a utilização da MM para o ensino apontam que essa combinação pode gerar bons frutos para o ensino e a aprendizagem da Matemática. Nesse ínterim, D’Ambrósio (1986) afirma que "os modelos matemáticos são formas de estudar e formalizar fenômenos do dia a dia. Através da Modelagem Matemática, o aluno se torna mais consciente da utilidade da matemática para resolver e analisar problemas do dia a dia” (p.17), possibilitando com isso, a percepção de que a Matemática pode ser manejada para compreender fenômenos com uso da criatividade em um processo investigativo e aplicado. Muitos autores divergem quanto as etapas de um processo de MM. Para fins didáticos, foram consideradas neste trabalho as cinco etapas propostas por Bassanezi (2011), a saber: experimentação (1), abstração (2), resolução (3), validação (4) e modificação (5).

A experimentação (1) é o momento no qual os dados serão compilados, ou seja, aqui tratamos do processo de coleta e organização dos dados. A partir de então, acontece a abstração (2), marcada pela formulação de hipóteses e criação de conjecturas para descrever o fenômeno estudado, entendendo a relação entre as variáveis consideradas para criação do modelo matemático. Na sequência, temos a resolução (3) do modelo encontrado. Esse por sua vez, é manipulado a partir de ferramentas que dependem do seu grau de complexidade de forma a encontrar uma interpretação matemática do modelo. A validação (4), busca saber se o modelo vai ser ou não aceito a partir dos testes de hipótese com os dados, ou seja, se verifica a validade do modelo em traduzir o fenômeno que foi modelado para então se chegar na quinta etapa, a modificação (5) que, enquanto última etapa do processo de modelagem, consiste em propor mudanças ao modelo encontrado com a finalidade de trazer ocasionais melhoras (Bassanezi, 2011). Nesse sentido, as fases da MM apresentadas pelo autor, vão ser de suma importante para o desenvolvimento do trabalho haja vista que a aula inovadora foi elaborada levando em consideração tal fundo teórico. 


\section{Metodologia}

Em termos metodológicos, a presente pesquisa possui natureza aplicada com procedimentos bibliográficos. Como classificações se fazem mediante a critérios, a seguir, serão justificadas as escolhas metodológicas. É importante destacar que a pesquisa em questão se divide em duas partes. A primeira delas se estabelece em um marco mais teórico em que é construído o referencial. A segunda parte é a elaboração de uma sequência de ensino (produzida com base na fase anterior) pensada para ser aplicada em condições reais de ensino.

A parte teórica é caracterizada pela presença da pesquisa bibliográfica que, segundo Gil (2017), "é desenvolvida com base em material já elaborado, constituído principalmente de livros e artigos científicos" (p. 44), ou seja, a partir das contribuições de vários autores e do confrontamento entre elas obteve-se as bases teórico-metodológicas que compõem o nosso referencial. Esses dados são obtidos por meio da leitura livros, artigos, dissertações, livros teses, sites e periódicos de nível nacional e internacional.

A partir desse referencial teórico, desenvolveu-se uma proposta de aula inovadora a ser implementada em condições reais de ensino, este momento caracteriza o presente trabalho como pesquisa aplicada, que segundo Silveira e Córdova (2009) objetiva "gerar conhecimentos para aplicação prática, dirigidos à solução de problemas específicos" (p. 35), nesse caso, sobretudo problemas relacionados ao ensino a aprendizagem que emergem das práticas educacionais desenvolvidas nas unidades escolares.

\section{Apresentação da Aula Inovadora}

A proposta de aula inovadora trata de uma sequência didática que propõe um estudo da Estatística a partir da abordagem MM para o desenvolvimento da literacia, do pensamento e do raciocínio estatístico. Vale ressaltar que sequência didática é entendida por Pereira et al. (2015) como "um conjunto de atividades conectadas, delineadas para ensinar um determinado conteúdo, passo a passo, estruturada e ajustada com os objetivos que estão sendo propostos pelo professor de modo a alcançar a aprendizagem de seus alunos" (p. 119). A aula inovadora foi elaborada com respaldo teórico nas concepções de MM apresentadas por Bassanezi (2011), por esse motivo, a sequência didática foi estruturada em três etapas: experimentação (1), criação e resolução de modelo (2), validação e modificação do modelo (3). Nas subseções que seguem, estão descritas cada uma dessas etapas.

\subsection{Etapa I: experimentação ( 90 min -2 aulas de 45 minutos)}

Esse momento se trata de uma aula expositiva dialogada em que será apresentado o site CoronavirusRN, plataforma utilizada para os fins didáticos da proposta de trabalho em questão. Dessa forma, os alunos serão direcionados a fazer um sobrevoo exploratório na plataforma, identificando os diferentes tipos de gráficos que estão ali presentes de forma a apontar seus elementos constitutivos. Tendo em vista a diversidade de gráficos na plataforma, a atenção deve ser concentrada nos seguintes gráficos:

a) Faixa etária dos pacientes com Covid-19: é um gráfico de coluna que apresenta a quantidade de pessoas infectadas dentro de determinado intervalo de idade, fazendo a divisão desses casos por sexo. Notadamente, um histograma.

b) Comorbidades: gráfico conhecido por nuvem de palavras ou nuvem de $\operatorname{tags}^{6}$. Ele exprime as principais doenças preexistentes dos pacientes diagnosticados com Covid-19 que foram a óbito. Essa informação é apresentada por meio de uma lista de palavras hierarquizadas visualmente.

\footnotetext{
${ }^{6}$ Em inglês, quer dizer etiquetas.
} 
c) Resultado dos testes: semelhante ao gráfico de pizza, no entanto, expressa o resultado acumulado dos testes de Covid-19 a partir de um quadrado e não de um círculo. O gráfico é dividido a partir das seguintes classes: positivo, negativos e outro.

d) Casos confirmados por dia: é um gráfico de colunas que apresenta o número total de casos notificados por dia.

e) Casos confirmados por grupo etário: é um gráfico de linhas que apresenta o histórico do número de casos que foram notificados diariamente divididos nas seguintes categorias: jovens, adultos e idosos.

f) Isolamento social - Rio Grande do Norte: expõe, por meio de um mapa do Rio grande do Norte, a taxa de mobilidade da população de cada município de forma que, os municípios assimilados com uma cor mais escura apresentam maior taxa de mobilidade e os que municípios com cor mais claras tem menor mobilidade.

A apresentação dos gráficos será permeada por perguntas de cunho investigativo ${ }^{7}$, de modo que os alunos sejam orientados a fazer suas próprias leituras a partir das informações apresentadas tomando conhecimento dos números da pandemia no Rio Grande do Norte. Espera-se que, com a familiarização, os alunos percebam a dinamicidade da plataforma e, por consequência, conheçam os diferentes gráficos que podem ser usados para veicular informações.

Para uma leitura adequada dos gráficos, é necessário conhecer o significado de alguns termos epidemiológicos comumente utilizados. Afinal, fazer a leitura dos gráficos sem compreendê-los, pode comprometer seriamente a interpretação dos dados. Isso visa estabelecer um ambiente de diálogo em que se possa discutir termos que a mídia e a comunidade científica vêm utilizando para divulgar e entender os números da pandemia (média móvel, taxa de transmissão, taxa de letalidade, número absoluto de mortes, mortes por milhão de habitantes, imunidade de rebanho) e, ao mesmo tempo, refletir sobre como acontece a coleta, organização e categorização desses dados.

\subsection{Etapa II: criação e resolução do modelo (135 min - 3 aulas de 45 minutos)}

Após a orientação realizada na etapa I, espera-se que os alunos estejam minimamente aptos a realizar a leitura e, por consequência, a interpretação de dados estatísticos. A segunda fase será trabalhada a partir do site RN+vacina, plataforma que traz as informações da vacinação no Rio Grande do Norte. O objetivo dessa etapa é fazer com que os alunos vejam nas informações apresentados por essa plataforma uma possibilidade de modelar esses dados, de forma que se perceba uma relação entre a quantidade de vacinados e o tempo de vacinação.

Essa etapa será realizada com pequenos grupos que devem agregar, no máximo, 4 componentes. Assim, após essa divisão, os alunos devem ser orientados por um material instrucional a ser entregue impresso pelo professor. Esse material compreende uma sequência de questionamentos que vão ajudar o aluno a perceber dados importantes de modo que direcionam o aluno à criação de um modelo. Após apresentar e visualizar os dados da vacinação no site RN+Vacina, a notícia contida na Figura 1 será disponibilizada para os alunos.

\footnotetext{
${ }^{7}$ A pergunta investigativa para Machado e Sansseron (2012) é "instrumento dialógico de estímulo à cadeia enunciativa. Sendo assim usado com propósito didático dentro da estória da sala de aula para traçar e acompanhar a construção de um significado e um conceito" (p. 31). Com isso, deve ser usada como propósito claro de guiar os estudantes no trabalho com as ideias científicas e dando suporte ao processo de internalização.
} 
Figura 1: Primeira vacinada contra Covid-19 no Rio Grande do Norte.

\begin{tabular}{|c|c|}
\hline 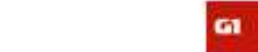 & RIO GRANDE DO NORTE $\underline{\boldsymbol{Q}}$ \\
\hline & $\begin{array}{l}\text { Técnica de Enfermagem é a } \\
\text { primeira pessoa a receber a vacina } \\
\text { contra a Covid-19 no RN: 'Chegou a } \\
\text { ser um sonho e hoje é esperança' }\end{array}$ \\
\hline & 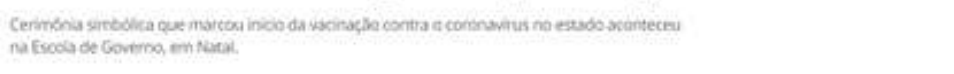 \\
\hline $\begin{array}{l}\text { Segundo a matéria } \\
\text { Escola de Governo }\end{array}$ & $\begin{array}{l}\text { cerimônia simbólica que marca o início da vacinação no } R N \text { aconteceu na } \\
\text { n Natal, na manhã desta terça (19). O ato contou com a presença da }\end{array}$ \\
\hline
\end{tabular}

Fonte: https://g1.globo.com/rn/rio-grande-do-norte/noticia/2021/01/19/tecnica-de-enfermagem-e-a-primeira-pessoa-a-receber-a-vacinacontra-a-covid-19-no-rn.ghtml.

Essa notícia foi escolhida por conter o dia em que a vacinação foi iniciada no RN, no entanto, qualquer outra fonte confiável que contenha essa informação pode ser usada para os fins didáticos da proposta. Vale ressaltar que, caso a escola disponha de internet e equipamentos informáticos, a pesquisa pode ser feita pelos próprios alunos. Com base na notícia eles deverão responder aos questionamentos presentes na atividade investigativa que será entregue impressa pelo professor.

A seguir, alguns dos questionamentos que podem ser apresentados com vistas a direcionar os alunos na atividade:

a) Segundo a notícia veiculada pela Figura 2, é possível determinar quantos dias se passaram desde o início da vacinação no estado do RN?

b) Segundo o site RN+Vacinas, quantas pessoas foram vacinadas até hoje com a primeira dose no Rio Grande do Norte?

c) Considerando as informações contidas nas Figura 1 e no site $\mathrm{RN}+\mathrm{vacina}$, qual foi a quantidade de pessoas vacinadas por dia com a primeira dose?

Depois disso, os alunos serão convidados a preencher uma tabela, proposta com o fito de organizar dados de modo que fosse possível perceber alguns aspectos importantes para a fase seguinte da atividade. Nesse caso, nos refeimos a Tabela 1 na sequência.

Tabela 1: explorando os números da vacinação

\begin{tabular}{|c|c|c|}
\hline PORCENTAGEM (\%) & $\begin{array}{c}\text { QUANTIDADE DE PESSOAS } \\
\text { VACINADAS }\end{array}$ & $\begin{array}{c}\text { TEMPO PARA } \\
\text { VACINAÇÃO }\end{array}$ \\
\hline 10 & 353416,5 & \\
\hline 50 & 1060249,5 & \\
\hline 90 & 1767082,5 & \\
\hline
\end{tabular}

Fonte: elaborada pelo autor (2021).

Organizados os dados na tabela e após a apreensão desses dados pelos alunos, o professor pode fazer perguntas de modo que eles vislumbrem a criação de um modelo a partir dos dados que eles se familiarizaram. Exemplos de perguntas que podem ser apresentadas, são: tendo em vista as grandezas quantidade de pessoas vacinadas e tempo para vacinação. É possível 
escrever uma expressão relacionando as duas grandezas? Qual relação de dependência você encontrou? Ou seja, quem depende de quem? Seria possível formular tal expressão? Ela precisa ser necessariamente algébrica?

Os dados disponibilizados devem permitir que o aluno determine o número médio de vacinados por dia, de modo que possa criar um modelo matemático que, no caso, representa uma função afim, do tipo $f(x)=a x+b$, em que $a$ é o número médio de vacinados por dia, $b$ é o total de pessoas já vacinadas até o momento, $x$ é o número de dias e $f(x)=y$ é a população do RN vacinada.

$\mathrm{Na}$ atividade, os alunos são orientados a criar um modelo que se aproxima de uma função afim. No entanto, variações podem surgir, como por exemplo, quando se considera a presença ou não de b na lei de formação. A verdade é que a depender do enfoque do professor, uma variedade de modelagens pode surgir, inclusive alguma que esteja associada a mais de uma sentença, que não precisa ser necessariamente numérica.

De posse do modelo, o material entregue segue com mais uma notícia (Figura 2) apresentada pelo Instituto Butantan do estado de São Paulo. Dessa vez, a respeito do "Projeto S", que vêm sendo empreendido pelo instituto na cidade de Serrana/SP, interior do estado.

Figura 2: projeto $\mathrm{S}$ em Serrana/SP.

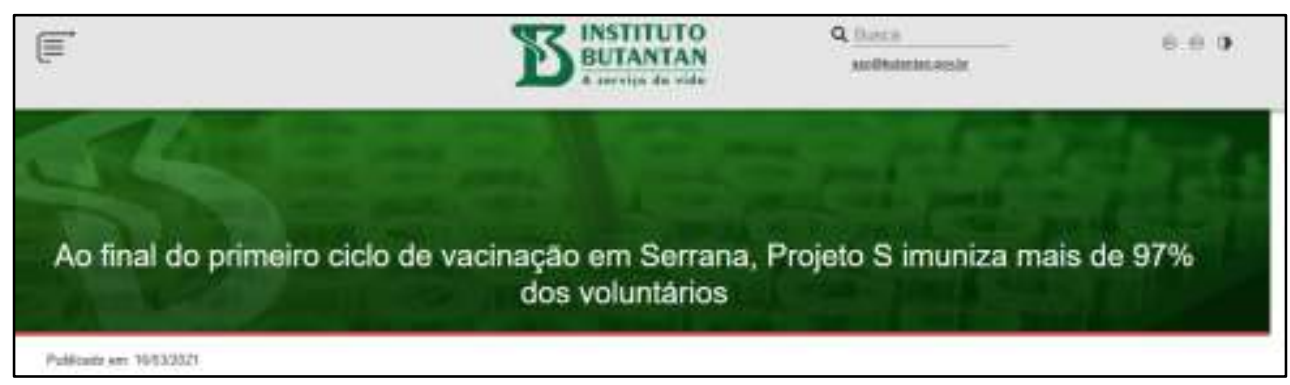

Fonte: https://butantan.gov.br/noticias/ao-final-do-primeiro-ciclo-de-vacinacao-em-serrana-projeto-s-imuniza-mais-de-97-dos-voluntarios.

A médica infectologista Natasha Nicos Ferreira é uma das responsáveis pelo projeto $\mathrm{S}$ e afirma na notícia que se cerca de $80 \%$ da população for imunizada, muito provavelmente será atingida a chamada imunidade coletiva, termo técnico usado para determinar a porcentagem mínima de imunizados para determinada população estar segura do vírus. A partir dessa evidência científica noticiada na página do Instituto Butantan, o aluno deverá utilizar o modelo que construiu a fim de obter as respostas para as seguintes perguntas:

1. Qual o tempo necessário para que toda a população do RN seja vacina?

2. Qual o tempo necessário para que a população do RN obtenha a imunidade coletiva?

Essas perguntas apresentadas serão um importante ponto de partida para que os modelos encontrados sejam utilizados pelos alunos de forma que respostas a problemas práticos possam ser determinadas. Espera-se que dessas perguntas possam emergir outros questionamentos que proporcionem uma compreensão com significado aos alunos.

\subsection{Etapa III: validação e modificação (45 minutos -1 aula)}

Na etapa II, cada grupo teve uma experiência com o seu modelo, a ideia é que ele utilize essa experiência de elaboração e elabore uma apresentação que contenha informações acerca do modelo elaborado e quais dificuldades surgiram durante o processo de modelagem. O grupo será convidado a expor a apresentação em sala de aula para que os colegas conheçam o trabalho dos colegas e, a partir disso, propor possíveis melhoras e adaptações a modelagem apresentada. Esse feedback dos alunos é

\footnotetext{
${ }^{8}$ O Instituto Butantan lançou em sua página do YouTube um documentário sobre o projeto S que, atualmente, tem 4 episódios. São detalhadas as fases de implementação do projeto e o seu desenvolvimento na cidade de Serrana/SP.
} 
importante pois permite que os resultados da etapa II sejam aprimorados, e posteriormente reformulados com vistas a encontrar um modelo mais fidedigno.

\section{Considerações Finais}

A Modelagem Matemática e a Educação Estatística são duas tendências que, cada vez mais, tem adentrado os espaços escolares. Vários pesquisadores e Educadores Matemáticos tem trabalhado em pesquisas em cada uma dessas áreas, no entanto, as possibilidades didáticas estão longe de se esgotar. Esse trabalho surge como mais uma possibilidade de trabalho dessas tendências, dessa vez de forma conjunta e harmônica.

Dessa maneira, valoriza-se o estudo de uma problemática presente no cotidiano do aluno e do professor, vinculando os conteúdos da cultura escolar àqueles presentes na vida do alunado. Essa aproximação envolvendo o aluno com a práxis social ao mesmo tempo em que se volta à formação de uma cidadania crítica permeada pelo pensamento matemático e estatístico. Espera-se que os alunos possam construir uma compreensão crítica dos dados apresentados nos gráficos, entendendo aspectos do seu contexto a partir do que a Estatística diz sobre ele.

Ressalta-se também a importância de trabalhar dados estatísticos vinculados a realidade para estimular o pensamento e garantir desenvolvimento da criticidade por parte do aluno. O uso dessas informações dentro de um ambiente permeado pela investigação permite a aproximação da Estatística com um contexto de Modelagem Matemática (MM), apontando para uma perspectiva de ensino da Estatística baseado no fomento das habilidades necessárias ao exercício da cidadania.

Além de pressupostos teóricos, necessários ao entendimento da prática docente, este artigo coloca à disposição da comunidade acadêmica e dos professores de Matemática uma proposta de aula que pode ser replicada em condições reis de ensino por professores de Matemática. Por esse motivo, cabe ressaltar a possibilidade de flexibilização e adaptação desta proposta. A realidade do professor que conhece seu alunado exige um dinamismo da prática pedagógica para superar as dificuldades de sua realidade escolar. Assim, apesar de terem sido usadas notícias específicas, um contexto específico e até mesmo uma forma de organização própria, a atividade pode ser adaptada com outras notícias ou mesmo outros contextos epidemiológicos.

Essa pesquisa reflete concepções sobre a Educação Estatística e viabiliza uma proposta para sala de aula. Esperamos que os apontamentos que apresentados, possam nortear a prática pedagógica de professores e possam servir como ponto de partida e uma oportunidade para a inserção de propostas como essa no dia a dia de sala de aula, de forma que os alunos possam construir conhecimento por meio de um aprendizado que seja carregado de significados, contextos e relações.

\section{Referências}

ASCOM/LAIS. (2020). Ecossistema tecnológico reúne informações sobre a UFRN. https://www.ufrn.br/imprensa/noticias/35006/ecossistema-tecnologico-reune-informacoes-sobre-a-covid-19.

Assis, L. B. (2015). Formação do usuário de Estatística pelo desenvolvimento da literacia estatística, do raciocínio estatístico e do pensamento estatístico através de atividades exploratórias. Dissertação (Mestrado profissional) - Curso de Mestrado em Educação Matemática, Instituto de Ciências Exatas, Universidade Federal de Juiz de Fora. p. 88.

Barbosa, J. C. (2004). Modelagem Matemática: O que é? Por que? Como? Veritati., (4), 73- 80

Bassanezi, R. C. (2011). Ensino-aprendizagem com Modelagem Matemática: uma nova estratégia. Contexto.

Bertone, A. M. A., Bassanezi, R. C., \& Jafelice, R. S. M. (2014). Modelagem Matemática. Ed. Universidade Federal de Uberlândia - UFU.

Brasil. (2018). Base Nacional Comum Curricular. Brasília: Ministério Da Educação.

Campos, C. R., Jacobini, O. R., Wodewotzki, L. M. L., \& Ferreira, D. (2011). Educação estatística no contexto da educação crítica. BOLEMA - Mathematics Education Bulletin, 24(39), 473-494.

D’ambrosio, U. (1986). Da realidade à ação reflexões sobre educação e matemática. Summus. 
Research, Society and Development, v. 11, n. 1, e49111125232, 2022

(CC BY 4.0) | ISSN 2525-3409 | DOI: http://dx.doi.org/10.33448/rsd-v11i1.25232

Freire, P. (2014). Educação e mudança. Paz e terra.

Gil, A. C. (2017). Como elaborar projetos de pesquisa. Atlas.

Lopes, P. C., \& Fernandes, E. (2014). Literacia, raciocínio e pensamento estatístico com robots. Quadrante, 23(2), 69-94.

Machado, V. F., \& Sasseron, L. H. (2012). As perguntas em aulas investigativas de ciências: a construção teórica de categorias. Revista Brasileira de Pesquisa em Educação em Ciências, 12(2), 29-44.

Mendonça, L. O., Lopes, C. E., \& Soares, E. (2013). Educação estatística em um ambiente de modelagem matemática nas aulas do ensino médio. Horizontes, $31(1), 9-19$

Meneguelli, L. (2017). Desenvolvimento de competências estatísticas no ensino médio por meio da modelagem matemática: analisando as diferentes representações. Dissertação (Mestrado em Educação em Ciências e Matemática) - Instituto Federal do Espírito Santo, p. 159.

Miguel, I. C., \& Natti, P. L. (2008). Uma Proposta de Modelagem Matemática Aplicada à Produção da Farinha de Trigo. Sertanópolis: UEL-Universidade Estadual de Londrina, p. 149

Oliveira, T. L. (2020). Quando as doenças viram números: as estatísticas da Covid-19. Fiocruz. http://coc.fiocruz.br/index.php/pt/todas-as-noticias/1809especial-covid-19-quando-as-doencas-viram-numeros-as-estatisticas-da-covid-19.html?tmpl=component\&print=1\&page=\#.X9hAgdhKjIX.

Pereira, A. C. C., Santiago, L. A., \& Morais, W. M. (2015). O uso de episódios históricos no ensino de matemática: uma sequência didática utilizando. In Pereira, A. C. C., \& Cedro, W. L. (Eds.). Educação matemática: diferentes contextos, diferentes abordagens (pp. 108-131). EDUECe.

Ponte, J. P., Brocardo, J., \& Oliveira, H. (2006). Investigação Matemática na sala de aula. Autêntica.

Silveira, D. T., \& Córdova, F. P. (2009). A pesquisa científica. In Gerhardt, T. E., \& Silveira, D. T. (org.). Métodos de Pesquisa (pp. 31-42). Editora da UFRGS.

Valentim, R. A. (2021). A relevância de um ecossistema tecnológico no enfrentamento à Covid-19 no Sistema Único de Saúde: o caso do Rio Grande do Norte. Ciência \& Saúde Coletiva, 26(1), 2035-2052. 